\title{
Exposure to Glycolytic Carbon Sources Reveals a Novel Layer of Regulation for the MalT Regulon
}

\author{
Sylvia A. Reimann and Alan J. Wolfe \\ Department of Microbiology and Immunology, Stritch School of Medicine, Loyola University Chicago, 2160 S. First Avenue, \\ Building 105, Maywood, IL 60153, USA \\ Correspondence should be addressed to Alan J. Wolfe, awolfe@lumc.edu
}

Received 16 March 2011; Revised 27 April 2011; Accepted 7 May 2011

Academic Editor: Haichun Gao

Copyright ( 12011 S. A. Reimann and A. J. Wolfe. This is an open access article distributed under the Creative Commons Attribution License, which permits unrestricted use, distribution, and reproduction in any medium, provided the original work is properly cited.

\begin{abstract}
Bacteria adapt to changing environments by means of tightly coordinated regulatory circuits. The use of synthetic lethality, a genetic phenomenon in which the combination of two nonlethal mutations causes cell death, facilitates identification and study of such circuitry. In this study, we show that the E. coli ompR malT ${ }^{\text {con }}$ double mutant exhibits a synthetic lethal phenotype that is environmentally conditional. MalT ${ }^{\mathrm{con}}$, the constitutively active form of the maltose system regulator MalT, causes elevated expression of the outer membrane porin LamB, which leads to death in the absence of the osmoregulator OmpR. However, the presence and metabolism of glycolytic carbon sources, such as sorbitol, promotes viability and unveils a novel layer of regulation within the complex circuitry that controls maltose transport and metabolism.
\end{abstract}

\section{Introduction}

Synthetic lethality, a phenomenon in which the combination of two nonlethal mutations causes death, is a powerful genetic tool that can, in an unbiased fashion, identify novel connections between cellular processes that function together to permit survival in a stressful environment. However, because the double mutant dies, investigating the process by which death occurs can be difficult. If, however, some permissive condition exists that permits survival of the double mutant, then the study of the death process is greatly facilitated, because genetic manipulations can be performed under permissive conditions and the consequences of those manipulations studied at nonpermissive conditions. Here, we explore one environmental condition (exposure to glycolytic carbon sources) that permits survival of the previously reported synthetic lethal mutant ompR malT con [1], which lacks the response regulator OmpR whilst harboring a constitutively active MalT ${ }^{\text {con }}$ protein.

As osmolality increases, the two-component response regulator OmpR becomes activated by the receipt of a phosphoryl group from its cognate sensor kinase EnvZ $[2,3]$. Upon phosphorylation, OmpR controls more than 100 genes associated with outer membrane biogenesis, osmoregulation, and envelope stress $[4,5]$.

MalT is the central regulator of all mal genes $[6,7]$ (Figure 1). The mal genes encode proteins involved in transport and metabolism of maltose and maltodextrins. The outer membrane porin LamB facilitates the uptake of maltose and maltodextrins into the periplasm, where these sugars are bound by the maltose-binding protein MalE and delivered to the $\mathrm{MalFGK}_{2}$ transporter [8]. Following transport into the cytoplasm, the sugars are metabolized [7]. MalT itself is activated by the maltose metabolite maltotriose and inhibited by MalK, MalY, Aes, and glucokinase [9-12]. The nucleoid proteins $\mathrm{H}-\mathrm{NS}$ and StpA positively regulate MalT translation $[13,14]$. malT transcription is activated by the cAMP-CRP complex, which renders it subject to catabolite repression $[15,16]$. Finally, Mlc represses malT transcription [17].

Under non-permissive conditions, the ompR malT con mutant displays a set of striking phenotypes. Colonies on plates are translucent and form papillae [1]. In liquid medium, the culture loses turbidity upon entry into late exponential phase [1], because the inner membrane disintegrates [18]. The main cause for these phenotypes is elevated 


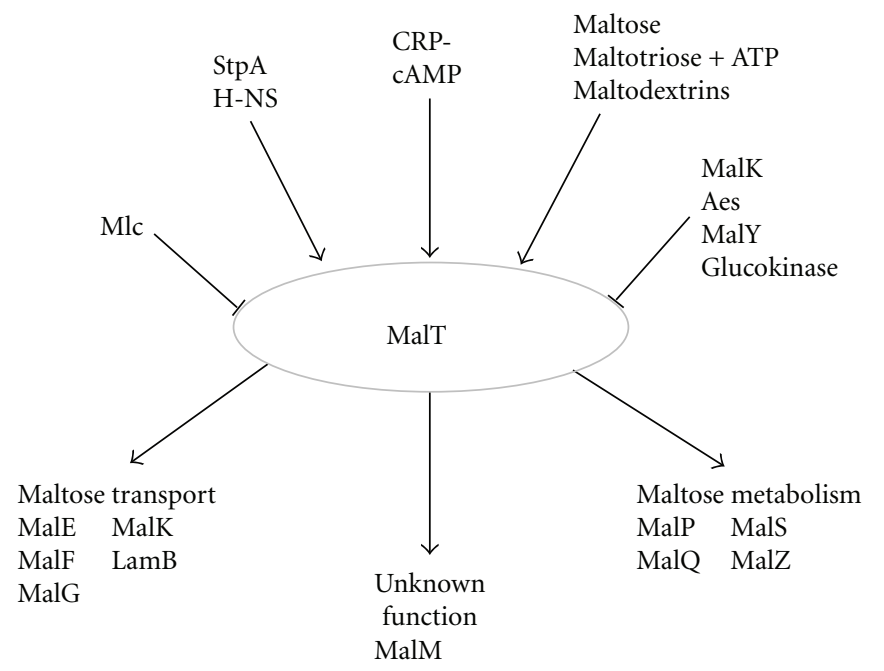

FIGURE 1: Regulation of MalT and its regulon. malT transcription is activated by the global regulator CRP-cAMP and repressed by Mlc. Translation of MalT is activated by H-NS and StpA. Activation of MalT activity can be attained by the binding of maltotriose, whereas it is inhibited by interaction with MalK, MalY, Aes, or glucokinase. Upon activation, MalT positively regulates expression of proteins that facilitate maltose uptake and metabolism.

LamB expression in the absence of OmpR: deletion of lamB permits survival [1], while genetic suppressors that reduce LamB expression also permit survival [18]. Similar to genetic suppressors, any environmental condition that reduces LamB levels should permit cell survival. For example, the ompR malT con mutant survives on minimal medium supplemented with glucose as the sole carbon source [1], almost certainly, because glucose causes catabolite repression of transcription from the malT and malK promoters and thus reduced expression of LamB $[15,19]$.

In this study, we identified an additional permissive environmental condition that supports survival of the $\operatorname{omp} R$ malT con mutant: the presence of noncatabolite repressing glycolytic carbon sources in the growth medium. Characterization of this condition allowed us to unveil a new regulatory layer of the MalT regulon. We hypothesize that this regulation requires metabolism of glycolysis-associated carbon sources.

\section{Materials and Methods}

2.1. Bacterial Strains, Bacteriophage, Transcriptional Fusions, and Plasmids. All bacterial strains used in this study are listed in Table 1. All strains evaluated were derivatives of E. coli AJW678 [20]. Derivatives were constructed by generalized transduction with P1vir, as described [21].

The transcription fusion malEp $\Delta 92-$ lac was a generous gift from Winfried Boos (Universität Konstanz, Germany) and was described previously [11].

The malT ${ }^{\text {con }}$ allele (malTc-1) used in this study was a generous gift from Linda Kenney (University of Illinois at Chicago, IL, USA). It harbors a T949A base substitution and encodes the MalT ${ }^{\mathrm{con}} \mathrm{W} 317 \mathrm{R}$ protein. Unless otherwise mentioned, deletion alleles were derived from the Keio collection [22].
2.2. Media and Growth Conditions. Because the ompR malT con mutant is conditionally lethal, cells were grown overnight under a permissive condition: $22^{\circ} \mathrm{C}$ in M63 minimal salts [21] with $22 \mathrm{mM}$ sorbitol as the sole carbon source and supplemented with $100 \mu \mathrm{g} \mathrm{mL} \mathrm{m}^{-1} \mathrm{~L}$-threonine, L-histidine, Lleucine, L-methionine, L-tryptophan, and $10 \mu \mathrm{g}$ thiamine $\mathrm{ml}^{-1}$. Whenever required, kanamycin $\left(40 \mu \mathrm{g} \mathrm{ml}^{-1}\right)$, chloramphenicol $\left(25 \mu \mathrm{g} \mathrm{ml}^{-1}\right)$, ampicillin $\left(100 \mu \mathrm{g} \mathrm{ml}^{-1}\right)$, or tetracycline $\left(15 \mu \mathrm{g} \mathrm{ml}^{-1}\right)$ was added.

For tests of lethality, an inoculum from the overnight culture was subcultured at $37^{\circ} \mathrm{C}$ in $\mathrm{LB}(1 \%(\mathrm{w} / \mathrm{v})$ tryptone, $0.5 \%(\mathrm{w} / \mathrm{v})$ yeast extract, $0.5 \%(\mathrm{w} / \mathrm{v}) \mathrm{NaCl})$. LB agar plates also contained $1.5 \%(\mathrm{w} / \mathrm{v})$ bacto agar. These growth conditions were considered non-permissive. Whenever required, carbon sources were added at a concentration of $22 \mathrm{mM}$. Cell growth was monitored spectrophotometrically (Beckman Instruments DU640) at $600 \mathrm{~nm}\left(\mathrm{OD}_{600}\right)$.

2.3. Promoter Activity Assays. To monitor malEp $\Delta 92-$ lac promoter activity, cells were grown aerobically with $250 \mathrm{rpm}$ agitation at $37^{\circ} \mathrm{C}$. At various time points during growth, $50 \mu \mathrm{L}$ aliquots were harvested and added to $50 \mu \mathrm{L}$ Allin-One $\beta$-galactosidase reagent (Pierce Biotechnology). $\beta$ galactosidase activity was determined quantitatively in a microtiter format, as described previously [23]. To avoid misleading results caused by lysing cells that spill $\beta$ galactosidase into the growth medium, we only considered $\beta$-galactosidase measurements before the onset of cell death.

2.4. Generation of Nonpolar Gene Deletions. To obtain nonpolar deletions, resistance cassettes were removed using flprecombinase, according to the previously described protocol [24]. 
TABLE 1: Strains, plasmids, and reporter fusions used in this study.

\begin{tabular}{|c|c|c|}
\hline & Relevant genotype & Reference \\
\hline AJW678 & thi-1thr-1(Am) leuB6 metF159(Am) rpsL136 lacX74 & {$[20]$} \\
\hline AJW2050 & AJW678 ompR::Tn10 & {$[44]$} \\
\hline AJW3098 & AJW678 ompR::Tn10 malT ${ }^{\mathrm{con}}$ T949A & {$[1]$} \\
\hline AJW3499 & AJW678 malT $T^{\text {con }} T 949 A$ & {$[1]$} \\
\hline AJW3732 & AJW678 ompR::Tn10 malT ${ }^{\mathrm{con}}(\mathrm{S} 358 \mathrm{I})$ & {$[1]$} \\
\hline AJW3733 & AJW678 ompR::Tn10 malT ${ }^{\mathrm{con}}(\mathrm{W} 317 \mathrm{P})$ & {$[1]$} \\
\hline AJW3734 & AJW678 ompR::Tn10 malT ${ }^{\text {con }}(\mathrm{R} 242 \mathrm{C})$ & {$[1]$} \\
\hline AJW3735 & AJW678 ompR::Tn10 malT ${ }^{\text {con }}(\mathrm{A} 244 \mathrm{E})$ & {$[1]$} \\
\hline AJW3736 & AJW678 ompR::Tn10 malT ${ }^{\mathrm{con}}$ (A236S) & {$[1]$} \\
\hline AJW3737 & AJW678 ompR::Tn10 malT ${ }^{\mathrm{con}}(\mathrm{A} 236 \mathrm{D})$ & {$[1]$} \\
\hline AJW3738 & AJW678 ompR::Tn10 malT ${ }^{\mathrm{con}}(\mathrm{P} 10 \mathrm{Q})$ & {$[1]$} \\
\hline AJW3739 & AJW678 ompR::Tn10 malT ${ }^{\mathrm{con}}$ (R242S) & {$[1]$} \\
\hline AJW3740 & AJW678 ompR::Tn10 malT ${ }^{\text {con }}$ (T38R) & {$[1]$} \\
\hline AJW3741 & AJW678 ompR::Tn10 malT ${ }^{\mathrm{con}}(\mathrm{S} 5 \mathrm{~L})$ & {$[1]$} \\
\hline AJW3888 & AJW678 ompR::Tn10 malT ${ }^{\text {con }}$ T949A $\Delta m a l Z:: K m$ & This study \\
\hline AJW3902 & AJW678 ompR::Tn10 malT ${ }^{\mathrm{con}}$ T949A $\Delta g l g P:: \mathrm{Km}$ & This study \\
\hline AJW3926 & AJW678 ompR::Tn10 malT ${ }^{\mathrm{con}}$ T949A $\Delta$ srlA::Km & This study \\
\hline AJW3927 & AJW678 ompR::Tn10 malT ${ }^{\mathrm{con}}$ T949A $\Delta s r l D:: \mathrm{Km}$ & This study \\
\hline AJW3936 & AJW678 ompR::Tn10 malT ${ }^{\mathrm{con}}$ T949A $\Delta m l c:: \mathrm{Km}$ & This study \\
\hline AJW3943 & AJW678 ompR::Tn10 malT con T949A $\Delta m a l Y:: K m$ & This study \\
\hline AJW3947 & AJW678 ompR::Tn10 malT ${ }^{\mathrm{con}}$ T949A $\Delta a e s:: \mathrm{Km}$ & This study \\
\hline AJW3967 & AJW678 ompR::Tn10 malT ${ }^{\mathrm{con}}$ T949A $\Delta$ malK::frt & This study \\
\hline AJW4023 & AJW678 ompR::Tn10 malT ${ }^{\mathrm{con}}$ T949A $\Delta h n s:: \mathrm{Km}$ & This study \\
\hline AJW4028 & AJW678 ompR::Tn10 malT ${ }^{\mathrm{con}} T 949 A \Delta s t p A:: \mathrm{Km}$ & This study \\
\hline AJW4197 & AJW678 ompR::Tn10 malT ${ }^{\mathrm{con}}$ T949A pstC::frt phoE::km & This study \\
\hline AJW4286 & AJW678 ompR::Tn10 malT ${ }^{\mathrm{con}}$ T949A $\Delta$ malK::frt $\triangle g l k:: \mathrm{Km}$ & This study \\
\hline \multicolumn{3}{|l|}{ Reporter fusions } \\
\hline malEp $\Delta 92-l a c$ & $\operatorname{trp}::[\text { KanR-malEp } \Delta 92-l a c]_{\mathrm{op}}$ & {$[30]$} \\
\hline
\end{tabular}

2.5. Outer Membrane Preparations. Outer membrane preparations were performed as described [25]. Outer membrane proteins were separated using 12\% SDS-PAGE containing 4.8 M urea and stained with Coomassie brilliant blue [26].

2.6. Semiquantitative RT-PCR. To compare malT transcript levels, cells were grown under the indicated conditions to an OD of 1. RNA was harvested using the RNeasy Mini kit (Quiagen). DNA contamination was removed by treatment with $5 \mathrm{U}$ of RQ1 RNase-free DNase (Promega) in 1x RQ1 DNase buffer for $1 \mathrm{~h}$ at $37^{\circ} \mathrm{C}$, followed by phenol-chloroform extraction and ethanol precipitation. The subsequent reverse transcription reaction was performed using the RevertAid First Strand cDNA Synthesis Kit (Fermentas). To exclude DNA contamination, we performed a mock cDNA reaction lacking reverse transcriptase. The resulting cDNA was diluted and PCR amplified in a reaction mixture containing $2 \mu \mathrm{L}$
cDNA product, $1 \times$ PCR buffer, $0.2 \mu \mathrm{M}$ dNTPs, $4 \mathrm{mM} \mathrm{MgCl}_{2}$, $0.5 \mu \mathrm{M}$ malT-specific primer malTfor2 (5'-ACTCAGCCCATAAGTCGGC-3' $), 0.5 \mu \mathrm{M}$ malT-specific primer malTrev2 (5'-CAAGACTTCAATCCCGCTAG-3'), and $1 \mathrm{U}$ Taq polymerase in a total volume of $25 \mu \mathrm{L}$. Amplification conditions were $95^{\circ} \mathrm{C}$ for $30 \mathrm{sec}, 54^{\circ} \mathrm{C}$ for $30 \mathrm{sec}$, and $72^{\circ} \mathrm{C}$ for $60 \mathrm{sec}(30$ cycles), followed by $72^{\circ} \mathrm{C}$ for $5 \mathrm{~min}$. The PCR products were subsequently analyzed on a $1 \%$ agarose gel.

\section{Results}

3.1. Metabolism of Glycolytic Carbon Sources Promotes Viability. We previously reported that the ompR malT ${ }^{\text {con }}$ double mutant (strain AJW3098, Table 1) exhibits a synthetic lethal phenotype caused by the increased expression of LamB [1].

Glucose catabolite represses mal transcription, and thus reduces LamB expression $[15,16]$. Therefore, it should not be 


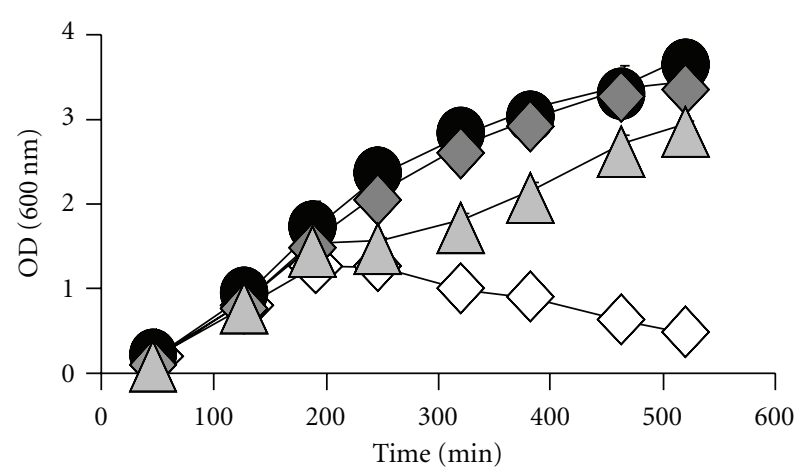

(a)

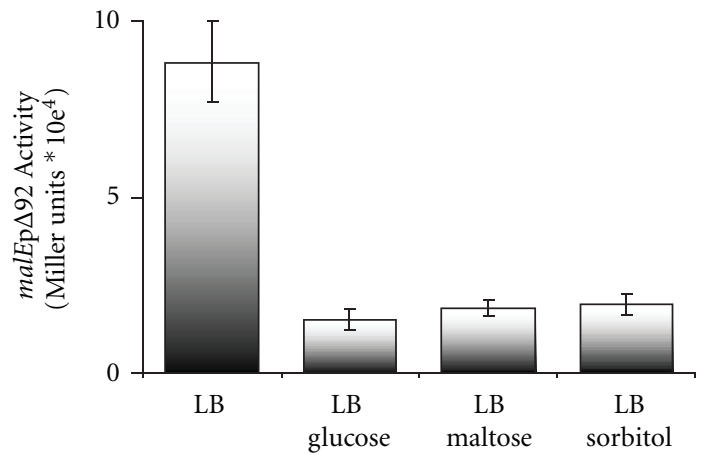

(b)

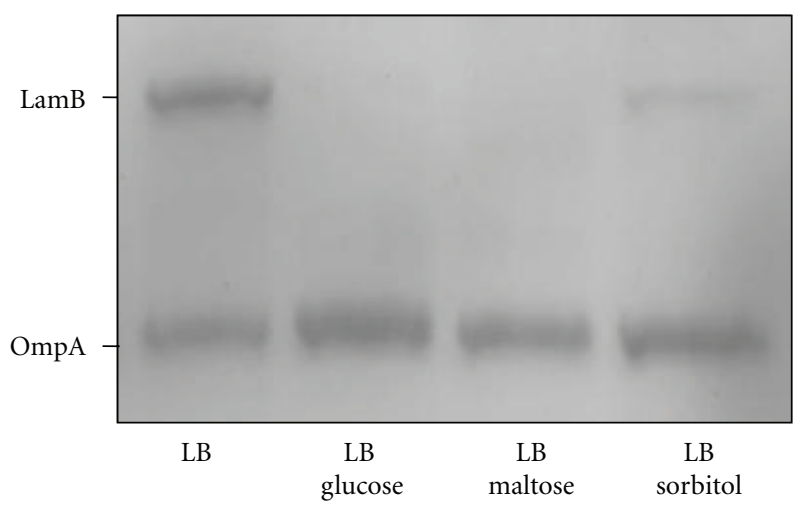

(c)

FIgURE 2: Effect of carbon sources on viability, mal gene transcription and LamB expression. (a) Growth curves of ompR malT ${ }^{\text {con }}$ mutants (strain AJW3098) grown in LB at $37^{\circ} \mathrm{C}$ without sugars (white diamonds) or supplemented with $22 \mathrm{mM}$ glucose (black circles), maltose (medium gray diamonds), or sorbitol (light gray triangles). Values represent the mean of triplicates. Error bars are only shown when greater than the symbol. (b) Effect of glycolytic carbon sources on mal gene transcription. $\beta$-galactoside activity was determined in ompR malT con mutants (strain AJW3098) carrying a malEp $\Delta 92$-lac reporter fusion. Cells were grown in LB without carbon source or LB supplemented with $22 \mathrm{mM}$ glucose, maltose, or sorbitol. Cells were harvested at an $\mathrm{OD}_{600}$ of 1 . Values represent the mean of triplicates. (c) Effect of glycolytic carbon sources on LamB expression. Addition of carbon sources reduces LamB levels in ompR malT con mutants as determined by outer membrane preparations. Cells were grown in $\mathrm{LB}$ at $37^{\circ} \mathrm{C}$ and harvested during late exponential phase. Gels were stained with Coomassie brilliant blue. Lane 1, LB no additional carbon source (LB); lane 2, LB $22 \mathrm{mM}$ glucose (LB glucose); lane 3, LB $22 \mathrm{mM}$ maltose (LB maltose); lane 4, LB $22 \mathrm{mM}$ sorbitol (LB sorbitol).

surprising that exposure to glucose promoted survival of the ompR malT ${ }^{\text {con }}$ mutant [1]. In contrast, the effect of maltose on mal gene expression can vary. Depending on expression or activity levels of the MalT protein, maltose can either enhance or inhibit MalT regulon expression [6, 27-29].

Here, we tested if exposure to maltose enhances or suppresses lethality of the ompR mal $T^{\mathrm{con}}$ mutant by growing it in $\mathrm{LB}$ at $37^{\circ} \mathrm{C}$ in the presence or absence of maltose and found that exposure to maltose suppressed death (Figure 2(a)). To test if this behavior is a general characteristic of malT ${ }^{\mathrm{con}}$ alleles, we tested if other mal $T^{\mathrm{con}}$ alleles behaved similarly. Several $\operatorname{ompR}$ malT ${ }^{\text {con }}$ double mutants harboring a set of representative mal $^{\text {con }}$ alleles (strains AJW3732-AJW3741) $[1,11]$ were grown in the presence or absence of maltose. As reported previously [1], each of these double mutants died in the absence of maltose. In contrast, they all survived in its presence (data not shown). We conclude that maltose can promote viability and that this behavior is a general characteristic of malT $T^{\text {con }}$ alleles.
Our finding that all the $\operatorname{ompR}$ malT ${ }^{\text {con }}$ double mutants survived when exposed to maltose, combined with our previous report that disruption of lamB permits survival [1], supports the earlier observation that maltose can reduce mal gene expression in cells carrying mal $T^{\mathrm{con}}$ alleles [29]. Thus, we asked if exposure to maltose reduces MalT regulon transcription. First, we monitored MalT regulon expression of an $\operatorname{ompR}$ malT ${ }^{\text {con }}$ double mutant carrying a transcriptional malEp $\Delta 92-l a c$ fusion [30]. We, then, directly monitored LamB expression using outer membrane preparations. Exposure of the ompR malT ${ }^{\text {con }}$ double mutant to maltose resulted in repressed malEp $\Delta 92$-lac transcription (Figure 2(b)) and reduced LamB expression (Figure 2(c) and Supplemental Figure 1(a) which is available at doi:10.1155/2011/107023). Similarly, the $m a l T^{\text {con }}$ single mutant displayed reduced LamB expression in the presence of maltose (Supplemental Figure 1(b)), indicating that this effect is independent of OmpR. In contrast, WT cells and the ompR single mutant showed an increase in LamB expression when maltose was 


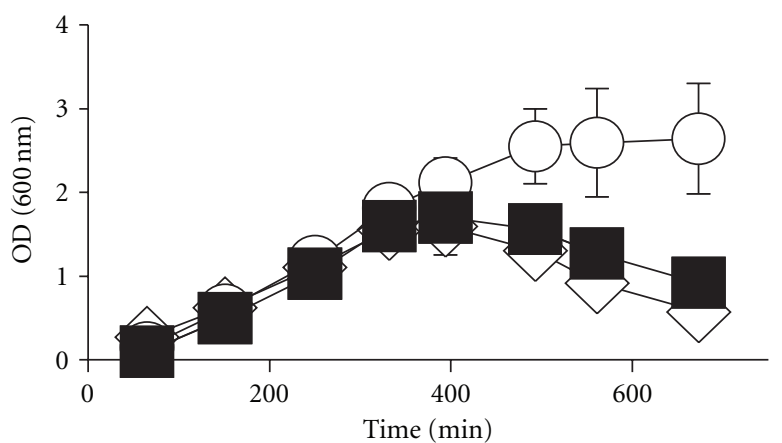

(a)

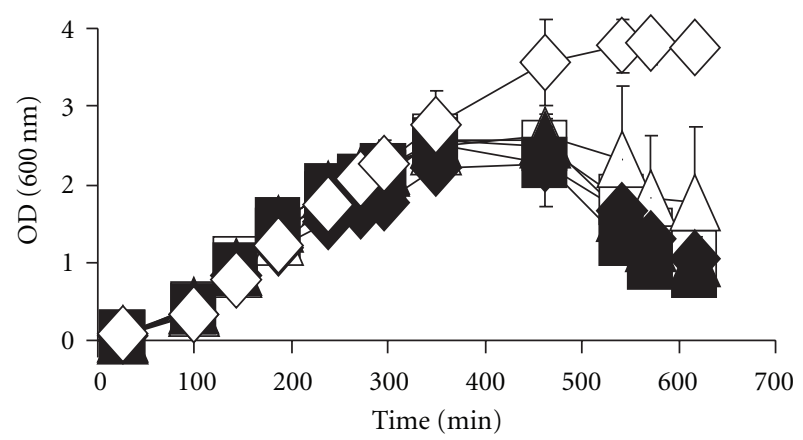

(b)

FIGURE 3: Effect of nonglycolytic carbon sources and sugar metabolism on viability. (a) Growth curves of $\operatorname{ompR}$ malT ${ }^{\text {con }}$ mutants (strain AJW3098) grown in $\mathrm{LB}$ at $37^{\circ} \mathrm{C}$ without additional carbon source (white diamonds) or supplemented with serine (white circles) or succinate (black squares). Values represent the mean of triplicates. Error bars are only shown when greater than the symbol. (b) Growth curves of ompR malT con, (strain AJW3098, diamonds), ompR malT ${ }^{{ }^{c o n}}$ srlA (strain AJW3926, squares) and ompR malT ${ }^{\text {con }} \operatorname{srlD}$ (strain AJW3927, triangles) mutants grown in LB at $37^{\circ} \mathrm{C}$. Black symbols, cells grown in LB without sorbitol; white symbols, cells grown in LB supplemented with $22 \mathrm{mM}$ sorbitol. Values represent the mean of triplicates. Error bars are only shown when greater than the symbol.

present (Supplemental Figure 2). We conclude that maltose supports survival of the $\operatorname{ompR}$ malT con double mutant by downregulating LamB expression.

The observation that maltose, a noncatabolite-repressing sugar, could reduce mal gene expression prompted us to ask if other noncatabolite-repressing sugars exert the same effect. We, therefore, grew the ompR malT con mutant in $\mathrm{LB}$ at $37^{\circ} \mathrm{C}$ in the presence or absence of diverse carbon sources. As expected, strong catabolite-repressing sugars (i.e., glucose, fructose, and mannitol) enabled survival (Figure 2(a) and Supplemental Table 1), whilst many noncatabolite-repressing carbon sources did not (Figure 3(a) and Supplemental Table 1). Surprisingly, some weaker or noncatabolite repressing carbon sources (i.e., sorbitol, serine, pyruvate, and mannose) promoted cell survival (Figures 2(a) and 3(a) and Supplemental Table 1). All the survivalsupporting carbon sources are metabolized via glycolysis, whilst all the nonsurvival supporting carbon sources are not. Thus, some glycolysis-associated mechanism, in addition to catabolite repression, must be able to promote cell survival.
To determine why carbon sources like sorbitol promoted cell survival, we first tested if sorbitol exerts its effect by influencing mal gene expression. We monitored both malEp $\Delta 92$ transcription and LamB expression in the ompR malT con double mutant supplemented with sorbitol and found them to be reduced (Figures 2(b) and 2(c)). We conclude that sorbitol promotes cell survival by reducing LamB expression.

Since sorbitol requires a transport mechanism that is different from maltose or glucose, $[7,8,31]$, we asked whether survival requires transport or metabolism of the sugar. We, therefore, constructed an $\operatorname{ompR}$ malT ${ }^{\text {con }}$ srlD triple mutant (strain AJW3927), which can transport sorbitol but not metabolize it. We also constructed an ompR malT con srlA triple mutant (strain AJW3926), which can neither transport nor metabolize sorbitol due to a polar effect of the $s r l A$ deletion on $s r l D$. Exposure to sorbitol did not permit survival of either mutant (Figure 3(b)). These results support the argument that the effect of sorbitol on the viability of the $\operatorname{ompR}$ mal $T^{\text {con }}$ double mutant requires metabolism of the sugar.

3.2. Sorbitol Promotes Survival through a Novel Mechanism. The mechanism by which sorbitol or a metabolite exerts its effect could require either the known regulators of the maltose regulon (Figure 1), or the outer membrane porin PhoE, which has been shown to promote viability when expressed at high levels [18]. Our aim was to test whether any of these factors are required for sorbitol-promoted survival. If none of these regulators is involved, we reasoned that sorbitol must operate through a currently unknown mechanism.

In a previous report, we found that the increased expression of the PhoB regulon member PhoE, an outer membrane porin, can promote viability of the ompR malT con double mutant [18]. Since sugar metabolism is known to de-repress the PhoB regulon [32], we tested whether sorbitol exerts its effect by increasing PhoE abundance in the outer membrane. However, the ompR malT ${ }^{\text {con }}$ pstC phoE mutant (strain AJW4197), which dies in LB [18], survived when we supplemented LB with sorbitol (data not shown). We conclude that sorbitol acts independently of PhoE.

To exert its effect on viability, products of sorbitol metabolism could act through a variety of regulators known to control malT expression or activity (Figure 1). For example, Mlc and CRP-cAMP affect malT transcription [15-17], HNS and StpA stimulate MalT translation [13, 14], and the binding of maltotriose, MalK, MalY, Aes, or glucokinase modulates MalT activity $[6,7,9-12,28]$.

The death of the ompR malT ${ }^{\text {con }}$ mlc triple mutant (strain AJW3936) in the absence of sorbitol and its survival when sorbitol was present (Supplemental Figure 3(a)) shows that sorbitol does not exert its effect by activating the transcriptional repressor Mlc (Figure 1) [17] or by increasing its concentration and thereby repressing malT transcription. That sorbitol did not influence malT transcription was confirmed by semiquantitative RT-PCR, which showed that exposure to sorbitol did not reduce malT ${ }^{\mathrm{con}}$ mRNA (Figure 4). Exposure to maltose also did not reduce malT $T^{\mathrm{con}} \mathrm{mRNA}$, confirming earlier reports that maltose affects $\mathrm{MalT}^{\mathrm{con}}$ activity rather than affecting malT $T^{\mathrm{con}}$ transcription $[6,28]$. Glucose, on the other hand, caused a reduction of malT ${ }^{\mathrm{con}} \mathrm{mRNA}$ (Figure 4), 


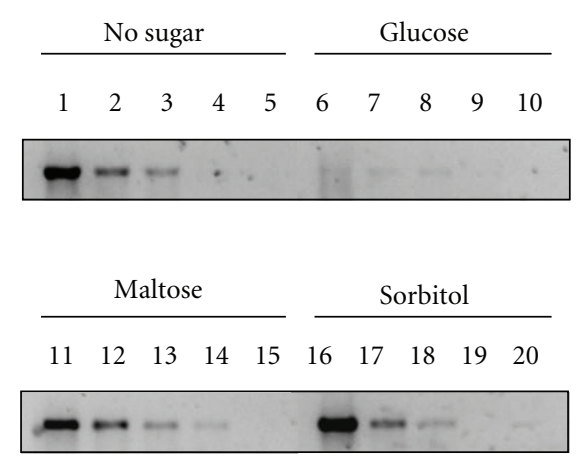

FIgURE 4: Effect of carbon sources on malT ${ }^{\text {con }}$ transcription. Semiquantitative RT-PCR of mal $T^{\mathrm{con}}$ in the $\operatorname{ompR}$ malT ${ }^{\text {con }}$ double mutant (strain AJW3098) grown under nonpermissive conditions in the absence (lane 1-5) or presence of glucose (lane 6-10), maltose (lane 11-15), or sorbitol (lane 16-20). PCR amplification was carried out with a dilution series of the cDNA: undiluted (lane 1, 6, 11 , and 16), $1: 10$ dilution (lane 2, 7, 12,17), 1:25 dilution (lane 3, 8,13 , and 18), $1: 125$ dilution (lane 4, 9, 14, 19), and mock control (lane 5, 10, 15, and 20).

which can be explained by its catabolite-repressing effect on malT transcription $[15,16]$. Thus, sorbitol permits viability of the ompR malT con double mutant by a mechanism that does not involve altered transcription of $m a l T^{\mathrm{con}}$.

Since sorbitol does not affect malT ${ }^{\mathrm{con}}$ transcription, we asked if it exerts its effect through any of the regulators that affect MalT ${ }^{\text {con }}$ activity. StpA is reported to exert a weak, activating effect on MalT regulon expression by modulating MalT translation [13]. Accordingly, we found that deletion of $\operatorname{stp} A$ in the ompR malT ${ }^{\text {con }}$ mutant (strain AJW4028) did not promote survival (Supplemental Figure 3(b)). We further determined that sorbitol did not exert its effect through StpA (Supplemental Figure 3(b)). Since deletion of hns in the $\operatorname{ompR}$ malT con double mutant promoted viability [18], we could not determine whether sorbitol exerts its effect through H-NS.

Next, we tested if sorbitol could affect MalT ${ }^{\text {con }}$ activity by altering maltotriose levels. We presumed that the excess carbon might be converted to glycogen and that the subsequent degradation of that glycogen might increase the intracellular maltotriose concentration, and cause endogenous induction of MalT (Figure 1) $[33,34]$. Whilst glycogen phosphorylase $(\mathrm{GlgP})$ is instrumental in the production of maltotriose from glycogen, maltodextrin glucosidase (MalZ) has been reported to remove maltotriose by hydrolyzing it to maltose and glucose [34]. Deletion of either malz or glgP in the ompR malT ${ }^{\text {con }}$ background (strains AJW3888 and AJW3902, respectively) did not rescue viability and did not diminish the ability of sorbitol to support growth (Figure 5). We conclude that the $o m p R$ malT ${ }^{\text {con }}$ mutant is largely insensitive to maltotriose and that sorbitol likely does not suppress lethality by altering maltotriose concentrations.

MalY, Aes, MalK, and glucokinase inhibit MalT activity (Figure 1) [9-12]. We, therefore, constructed the triple mutants ompR malT ${ }^{\text {con }}$ malY (strain AJW3943) and ompR

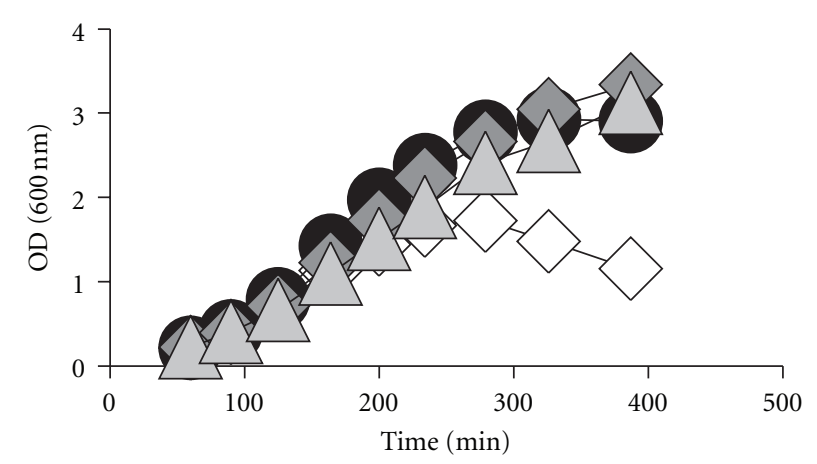

(a)

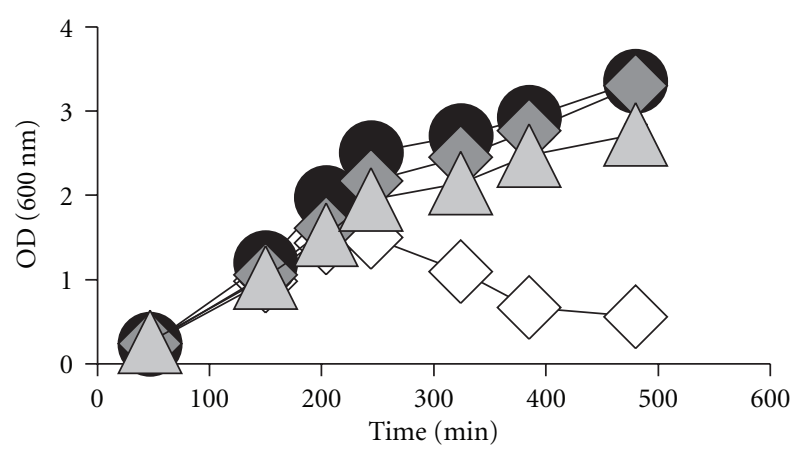

(b)

FIGURE 5: Effect of maltotriose on viability. (a) Growth curves of the ompR malT ${ }^{\text {con }} g \operatorname{lgP}$ mutant (strain AJW3902) grown in LB at $37^{\circ} \mathrm{C}$ without sugars (white diamonds) or supplemented with glucose (black circles), maltose (dark gray diamonds), or sorbitol (light gray triangles). Values represent the mean of triplicates. Error bars are only shown when greater than the symbol. (b) Growth curves of the ompR malT con malZ mutant (strain AJW3888) grown in LB at $37^{\circ} \mathrm{C}$ without sugars (white diamonds) or supplemented with glucose (black circles), maltose (dark gray diamonds), or sorbitol (light gray triangles). Values represent the mean of triplicates. Error bars are only shown when greater than the symbol.

malT con aes (strain AJW3947), and an ompR malT con malK triple mutant carrying a nonpolar malK allele (strain AJW3967) to avoid disruption of LamB expression. We further constructed an ompR malT ${ }^{\text {con }}$ malK glk quadruple mutant (strain AJW4286). We monitored growth of the first two mutants under non-permissive conditions in the presence or absence of glucose, maltose, or sorbitol. Since null mutations of malK and/or glk cause defects in the importation or metabolism of maltose, mutants carrying these alleles were only grown in the presence of glucose or sorbitol. In response to all tested sugars, the mutants survived (Supplemental Figures 4(a)-4(d)). Thus, none of the sugars, including sorbitol, act through MalY, Aes, MalK, or glucokinase.

Since sorbitol-dependent survival of the ompR malT con mutant depends on none of the known regulatory mechanisms, we hypothesize that a novel regulatory mechanism exists, which involves posttranscriptional modulation of MalT activity. 


\section{Discussion}

4.1. Glycolysis Provides a New Layer of Regulation to the Maltose System. A highly complex network integrates numerous diverse signals to precisely regulate the expression and function of the maltose transport and metabolism system $[6,7,35]$ (Figure 1). We now hypothesize that an additional regulatory layer exists that involves glycolysis. We base this hypothesis on the observation that the synthetic lethality of the ompR malT con mutant can be inhibited by growth in the presence of several glycolysis-associated carbon sources (Figures 2(a) and 3(a) and Supplemental Table 1). That a sugar like glucose or fructose can inhibit lethality is easily explained by its capacity to catabolite-repress malTp and malKp transcription and, hence, limit LamB expression [15, 16]. That sugars like maltose and sorbitol also can inhibit lethality, however, is both surprising and telling.

4.2. Inhibition by Maltose. Depending on MalT levels or MalT activity, the effect of maltose on MalT regulon expression can vary. In cells expressing WT MalT in large excess, it is reported that exposure to maltose causes a slight reduction ( $\sim 2$-fold) in mal gene expression [29]. However, in cells moderately overexpressing WT MalT, exposure to maltose has been reported to induce MalT regulon expression [29]. The same is true when cells express WT MalT from the endogenous gene $[6,28]$. We confirmed this observation by showing that LamB levels increase in WT cells and ompR mutants when maltose is present (Supplemental Figure 2). If the same were true of cells that carry the malT ${ }^{\text {con }}$ allele, then the resulting increase in LamB levels would be expected to lead to an even more premature death of the ompR malT con double mutant. Instead, maltose reduced malE transcription and LamB expression, and thus permitted survival (Figures 2(b) and 2(c) and Supplemental Figure 1).

In cells carrying malT ${ }^{\text {con }}$ alleles, exposure to maltose has been reported to induce expression at the malE promoter, with the notable exception of highly constitutive MalT ${ }^{\text {con }}$ proteins [27, 29]. In contrast, we found that exposure to maltose causes reduced malE promoter activity in the ompR malT con double mutant (Figure 2(b)) and reduced LamB levels in both the malT ${ }^{\mathrm{con}}$ single and ompR malT con double mutants (Figure 2(c) and Supplemental Figure 1), permitting the ompR malT con double mutant to survive. Since survival was observed in all $10 \mathrm{ompR}$ malT ${ }^{\text {con }}$ mutants tested, representing each location cluster and inhibition class [11], it is likely that the observed inhibitory response is a general characteristic of MalT ${ }^{\text {con }}$ proteins. The discrepancy between this and the previous reports could be due to utilization of different strain backgrounds (MC4100 versus AJW678) or of different media (minimal medium with glycerol as the base carbon source and supplemented with maltose versus LB supplemented with maltose).

In a previous study, exposure to maltose in the context of high expression of MalT ${ }^{\mathrm{WT}}$ caused a moderate 2-fold reduction of malE transcription [29]. To explain this result, a model was proposed in which overproduction of MalTWT results in a large number of MalT ${ }^{\mathrm{WT}}$ oligomers that substitute for CRP. This would result in formation at the malEp and malkp promoters of a less active nucleoprotein complex containing only MalT [36]. A further development of this model proposed unlimited aggregation of MalT to be responsible for the inhibition of malEp transcription at high concentrations of MalT [37]. For these models to explain our observations, the native gene would have to express enough MalT ${ }^{\text {con }}$ to permit successful competition with CRP for DNA binding. Furthermore, those constitutively active proteins would have to become more active in response to maltose. Finally, the combination would have to be able to exert a 2-fold larger effect (4-fold inhibition) than did the wild-type protein expressed from a multicopy plasmid (2fold inhibition). We think it is more likely that maltose acts upon MalT ${ }^{\text {con }}$ in a manner similar to that of sorbitol.

4.3. Inhibition by Sorbitol. Sorbitol has never been reported to influence the maltose system or inhibit MalT regulon transcription; thus, the inhibitory mechanism through which it operates must be novel. With the notable exceptions of $\mathrm{H}$ NS and the CRP-cAMP complex, we tested the involvement of each known MalT regulator (Figure 1) and found that none are required (Figures 4 and 5 and Supplemental Figures 3 and 4). We ruled out the CRP-cAMP complex, because sorbitol causes only weak catabolite repression $[8,38]$. This argument is further supported by the observation that exposure to sorbitol did not alter malT $^{\text {con }}$ mRNA levels (Figure 4). We also excluded H-NS, since, to our knowledge, the global regulator has never been reported to respond to glycolysis. Thus, we hypothesize that sorbitol exerts its effect on mal gene transcription through a novel mechanism that is independent of the currently reported regulators and signals.

Our studies show that the lethal phentoype caused by the MalTcon protein used in this study is insensitive to both the inducer maltotriose (Figure 5) and the inhibitor MalK $[1,39]$ (data not shown). Although MalK can exert a small effect on the activity of this MalTcon protein, an observation made when we tested media that does not contain the MalT inducer trehalose, this small effect was insufficient to influence the lethal phenotype (Reimann and Wolfe, unpublished data). Thus, stripped of the two primary layers of regulation provided by maltotriose and MalK, the ompR malT ${ }^{\text {con }}$ double mutant exposes an otherwise undetectable layer of regulation. That sorbitol must be metabolized to inhibit MalT regulon transcription (Figure 3(b)) suggests the existence of a central metabolite that modulates MalT regulon expression.

The identity of this central metabolite remains unknown. However, recent reports that CRP and other transcription factors can become acetylated [40-42] coupled with the knowledge that the protein deacetylase CobB depends on $\mathrm{NAD}^{+}$for its function [43] raises the exciting possibility that increased glycolytic flux due to metabolism of the excess sorbitol results in acetylation of CRP, MalT, or some other component of the nucleoprotein complex that modulates malE and malK transcription, resulting in inhibition and thus survival.

The concept of a glycolytic metabolite opens up the possibility that maltose, glucose, fructose, and other glycolytic 
carbon sources could work through the same mechanism. In the case of glucose and fructose, however, the effect is normally concealed by their strong catabolite-repressing effect. Likewise, in cells harboring a MalT ${ }^{W T}$ protein, the strong regulatory effects of maltotriose and MalK would normally counterbalance the regulatory effect of maltose metabolism.

\section{Acknowledgments}

The authors thank Winfried Boos, Tom Silhavy, Linda Kenney, and the National Institute for Genetics (Japan) for providing strains, reporter fusions, plasmids and phage, Bozena Zemaitaitis for performing experiments with the ompR malT con malK glk mutant, Karen Visick for fruitful discussions, members of the Wolfe and Visick labs for critical reading of the paper, and the National Institute of General Medical Sciences (GM066130) and the Loyola University Potts Foundation (LU\#11200) for funding.

\section{References}

[1] S. A. Reimann and A. J. Wolfe, "A critical process controlled by MalT and OmpR is revealed through synthetic lethality," Journal of Bacteriology, vol. 191, no. 16, pp. 5320-5324, 2009.

[2] M. M. Igo, J. M. Slauch, and T. J. Silhavy, "Signal transduction in bacteria: kinases that control gene expression," New Biologist, vol. 2, no. 1, pp. 5-9, 1990.

[3] T. Mizuno, A. Shinkai, K. Matsui, and S. Mizushima, "Osmoregulatory expression of porin genes in Escherichia coli: a comparative study on strains B and K-12," FEMS Microbiology Letters, vol. 68, no. 3, pp. 289-293, 1990.

[4] T. Oshima, H. Aiba, Y. Masuda et al., "Transcriptome analysis of all two-component regulatory system mutants of Escherichia coli K-12," Molecular Microbiology, vol. 46, no. 1, pp. 281-291, 2002.

[5] B. M. Pruss, C. A. Besemann, A. M. Denton, and A. J. Wolfe, "A complex transcription network controls the early stages of biofilm development by Escherichia coli," Journal of Bacteriology, vol. 188, no. 11, pp. 3731-3739, 2006.

[6] W. Boos and A. Bohm, "Learning new tricks from an old dog: MalT of the Escherichia coli maltose system is part of a complex regulatory network," Trends in Genetics, vol. 16, no. 9, pp. 404409, 2000 .

[7] W. Boos and H. A. Shuman, "Maltose/maltodextrin system of Escherichia coli: transport, metabolism, and regulation," Microbiology and Molecular Biology Reviews, vol. 62, no. 1, pp. 204-229, 1998.

[8] M. Ehrmann, R. Ehrle, E. Hofmann, W. Boos, and A. Schlosser, "The ABC maltose transporter," Molecular Microbiology, vol. 29, no. 3, pp. 685-694, 1998.

[9] R. Peist, A. Koch, P. Bolek, S. Sewitz, T. Kolbus, and W. Boos, "Characterization of the aes gene of Escherichia coli encoding an enzyme with esterase activity," Journal of Bacteriology, vol. 179, no. 24, pp. 7679-7686, 1997.

[10] N. Joly, A. Bohm, W. Boos, and E. Richet, "MalK, the ATPbinding cassette component of the Escherichia coli maltodextrin transporter, inhibits the transcriptional activator MalT by antagonizing inducer binding," Journal of Biological Chemistry, vol. 279, no. 32, pp. 33123-33130, 2004.
[11] A. Schlegel, O. Danot, E. Richet, T. Ferenci, and W. Boos, "The N terminus of the Escherichia coli transcription activator MalT is the domain of interaction with MalY," Journal of Bacteriology, vol. 184, no. 11, pp. 3069-3077, 2002.

[12] C. Lengsfeld, S. Schonert, R. Dippel, and W. Boos, "Glucoseand glucokinase-controlled mal gene expression in Escherichia coli," Journal of Bacteriology, vol. 191, no. 3, pp. 701-712, 2009.

[13] J. Johansson, B. Dagberg, E. Richet, and B. E. Uhlin, "H-NS and StpA proteins stimulate expression of the maltose regulon in Escherichia coli," Journal of Bacteriology, vol. 180, no. 23, pp. 6117-6125, 1998.

[14] H. S. Park, Y. Ostberg, J. Johansson, E. G. H. Wagner, and B. E. Uhlin, "Novel role for a bacterial nucleoid protein in translation of mRNAs with suboptimal ribosome-binding sites," Genes and Development, vol. 24, no. 13, pp. 1345-1350, 2010.

[15] C. Chapon, "Role of the catabolite activator protein in the expression of the maltose regulon of Escherichia coli," Annales de Microbiologie, vol. 133, no. 1, pp. 77-80, 1982.

[16] C. Chapon and A. Kolb, "Action of CAP on the malT promoter in vitro," Journal of Bacteriology, vol. 156, no. 3, pp. 1135-1143, 1983.

[17] K. Decker, J. Plumbridge, and W. Boos, "Negative transcriptional regulation of a positive regulator: the expression of MalT, encoding the transcriptional activator of the maltose regulon of Escherichia coli, is negatively controlled by Mlc," Molecular Microbiology, vol. 27, no. 2, pp. 381-390, 1998.

[18] S. A. Reimann and A. J. Wolfe, "Constitutive expression of the maltoporin LamB in the absence of OmpR damages the cell envelope," Journal of Bacteriology, vol. 193, no. 4, pp. 842-853, 2011.

[19] E. Richet, D. Vidal-Ingigliardi, and O. Raibaud, "A new mechanism for coactivation of transcription initiation: repositioning of an activator triggered by the binding of a second activator," Cell, vol. 66, no. 6, pp. 1185-1195, 1991.

[20] S. Kumari, C. M. Beatty, D. Browning et al., "Regulation of acetyl coenzyme A synthetase in Escherichia coli," Journal of Bacteriology, vol. 182, no. 15, pp. 4173-4179, 2000.

[21] T. J. Silhavy, M. L. Berman, and L. W. Enquist, Experiments with Gene Fusions, Cold Spring Harbor Laboratory Press, Cold Spring Harbor, NY, USA, 1984.

[22] M. Baba, T. Ara, M. Hasegawa et al., "Construction of Escherichia coli K-12 in-frame, single-gene knockout mutants: the Keio collection," Molecular Systems Biology, vol. 2, article 2006.0008, 2006.

[23] C. M. Beatty, D. Browning, S. J. W. Busby, and A. J. Wolfe, "Cyclic AMP receptor protein-dependent activation of the Escherichia coliacsP2 promoter by a synergistic class III mechanism," Journal of Bacteriology, vol. 185, no. 17, pp. 5148-5157, 2003.

[24] K. A. Datsenko and B. L. Wanner, "One-step inactivation of chromosomal genes in Escherichia coli K-12 using PCR products," Proceedings of the National Academy of Sciences of the United States of America, vol. 97, no. 12, pp. 6640-6645, 2000.

[25] R. Morona and P. R. Reeves, "The tolC locus of Escherichia coli affects the expression of three major outer membrane proteins," Journal of Bacteriology, vol. 150, no. 3, pp. 10161023, 1982.

[26] J. Sambrook, E. F. Fritsch, and T. Maniatis, Molecular Cloning-A Laboratroy Manual, Cold Spring Harbor Laboratory Press, Cold Spring Harbor, NY, USA, 1989.

[27] B. Dardonville and O. Raibaud, "Characterization of malT mutants that constitutively activate the maltose regulon of Escherichia coli," Journal of Bacteriology, vol. 172, no. 4, pp. 1846-1852, 1990. 
[28] O. Raibaud and E. Richet, "Maltotriose is the inducer of the maltose regulon of Escherichia coli," Journal of Bacteriology, vol. 169, no. 7, pp. 3059-3061, 1987.

[29] M. Schwartz, "The maltose regulon," in Escherichia coli and Salmonella typhimurium. Cellular and Molecular Biology, F. C. Neidhardt and J. L. Ingraham, Eds., American Society for Microbiology, Washington, DC, USA, 1987.

[30] E. Richet, "On the role of the multiple regulatory elements involved in the activation of the Escherichia colimalEp promoter," Journal of Molecular Biology, vol. 264, no. 5, pp. 852-862, 1996.

[31] F. C. Grenier, E. B. Waygood, and M. H. Saier Jr., "The bacterial phosphotransferase system: kinetic characterization of the glucose, mannitol, glucitol, and $\mathrm{N}$-acetylglucosamine systems," Journal of Cellular Biochemistry, vol. 31, no. 2, pp. 97-105, 1986.

[32] A. Hartmann and W. Boos, "Mutations in phoB, the positive gene activator of the pho regulon in Escherichia coli, affect the carbohydrate phenotype on MacConkey indicator plates," Research in Microbiology, vol. 144, no. 4, pp. 285-293, 1993.

[33] K. Decker, R. Peist, J. Reidl, M. Kossmann, B. C. Brand, and W. Boos, "Maltose and maltotriose can be formed endogenously in Escherichia coli from glucose and glucose-1-phosphate independently of enzymes of the maltose system," Journal of Bacteriology, vol. 175, no. 17, pp. 5655-5665, 1993.

[34] R. Dippel, T. Bergmiller, A. Bohm, and W. Boos, "The maltodextrin system of Escherichia coli: glycogen-derived endogenous induction and osmoregulation," Journal of Bacteriology, vol. 187, no. 24, pp. 8332-8339, 2005.

[35] A. Schlegel, A. Bohm, S. J. Lee, R. Peist, K. Decker, and W. Boos, "Network regulation of the Escherichia coli maltose system," Journal of Molecular Microbiology and Biotechnology, vol. 4, no. 3, pp. 301-307, 2002.

[36] O. Raibaud, D. Vidal-Ingigliardi, and E. Richet, "A complex nucleoprotein structure involved in activation of transcription of two divergent Escherichia coli promoters," Journal of Molecular Biology, vol. 205, no. 3, pp. 471-485, 1989.

[37] V. Schreiber and E. Richet, "Self-association of the Escherichia coli transcription activator MalT in the presence of maltotriose and ATP," Journal of Biological Chemistry, vol. 274, no. 47, pp. 33220-33226, 1999.

[38] J. W. Lengeler and E. Lin, "Reversal of the mannitol-sorbitol diauxie in Escherichia coli," Journal of Bacteriology, vol. 112, no. 2, pp. 840-848, 1972.

[39] M. Reyes and H. A. Shuman, "Overproduction of MalK protein prevents expression of the Escherichia coli mal regulon," Journal of Bacteriology, vol. 170, no. 10, pp. 4598-4602, 1988.

[40] S. Thao, C.-S. Chen, H. Zhu, and J. C. Escalante-Semerena, "Nepsilon-lysine acetylation of a bacterial transcription factor inhibits its DNA-binding activity," PLoS One, vol. 5, no. 12, article, 2010.

[41] B. J. Yu, J. A. Kim, J. H. Moon, S. E. Ryu, and J. G. Pan, "The diversity of lysine-acetylated proteins in Escherichia coli," Journal of Microbiology and Biotechnology, vol. 18, no. 9, pp. 1529-1536, 2008.

[42] J. Zhang, R. W. Sprung, J. Pei et al., "Lysine acetylation is a highly abundant and evolutionarily conserved modification in Escherichia coli," Molecular and Cellular Proteomics, vol. 8, no. 2, pp. 215-225, 2009.
[43] V. J. Starai, I. Celic, R. N. Cole, J. D. Boeke, and J. C. Escalante-Semerena, "Sir2-dependent activation of acetylCoA synthetase by deacetylation of active lysine," Science, vol. 298, no. 5602, pp. 2390-2392, 2002.

[44] M. Berman, L. Enquist, and T. J. Silhavy, Advanced Bacterial Genetics, Cold Spring Harbor Lab Press, Cold Spring Harbor, NY, USA, 1981. 

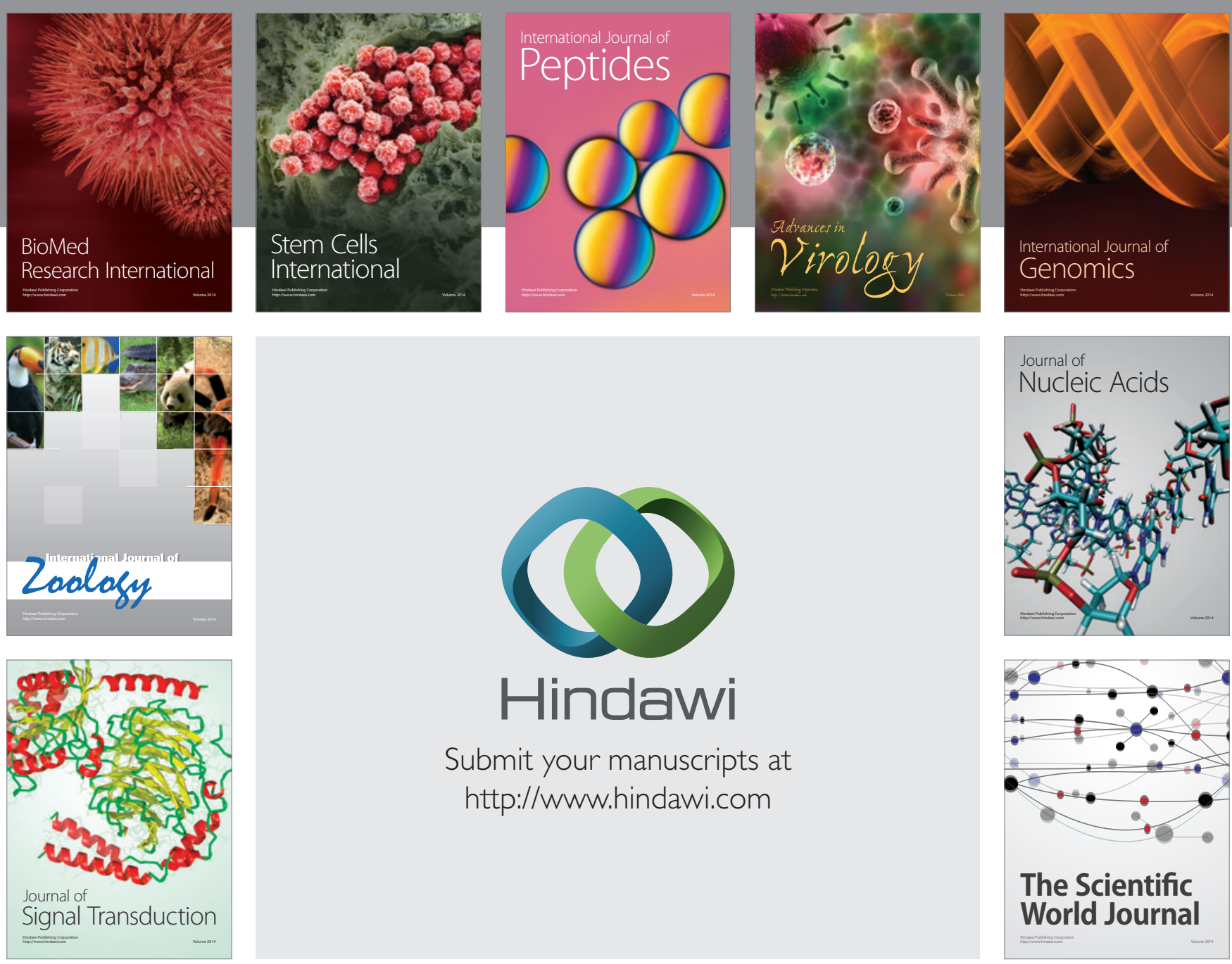

Submit your manuscripts at

http://www.hindawi.com
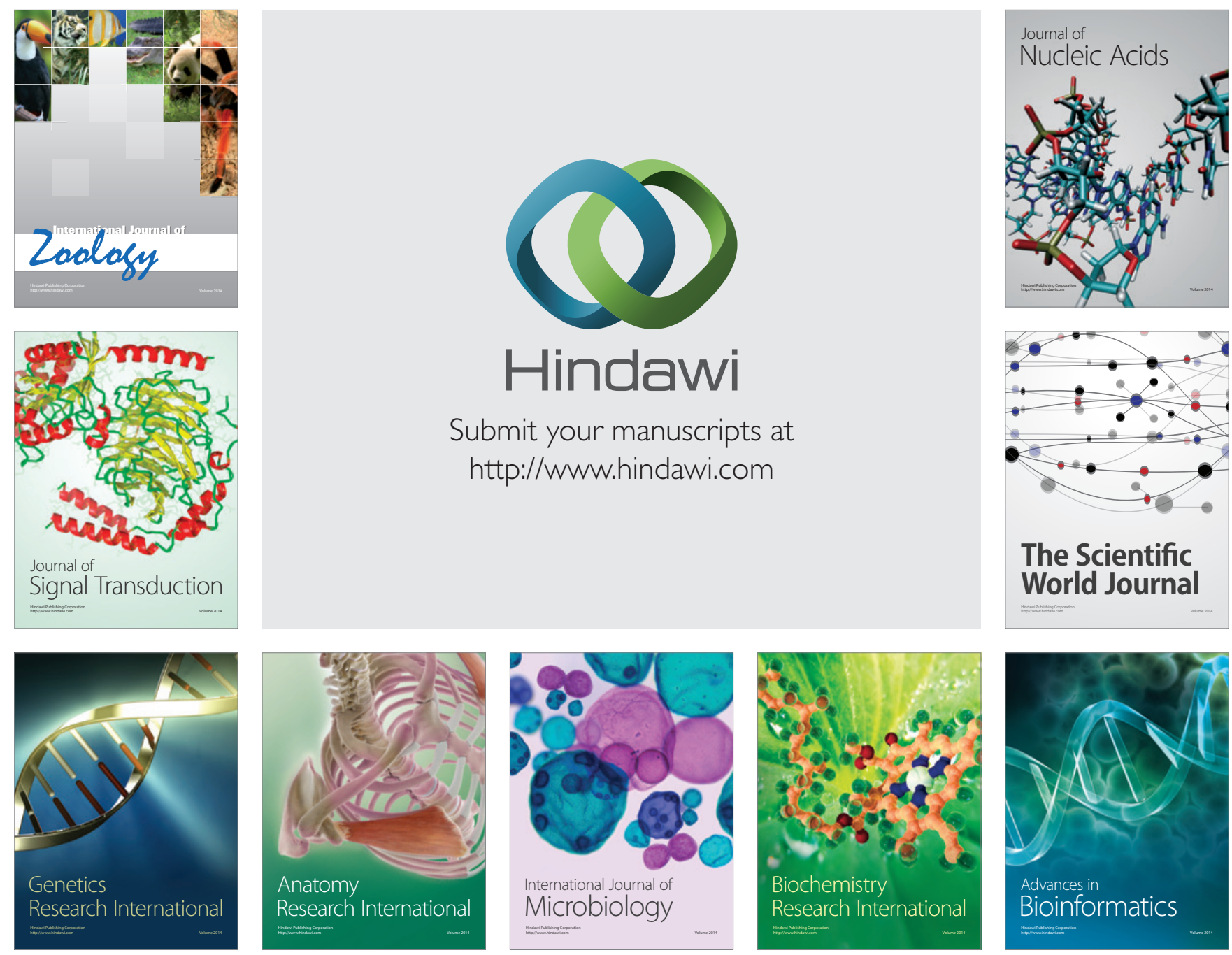

The Scientific World Journal
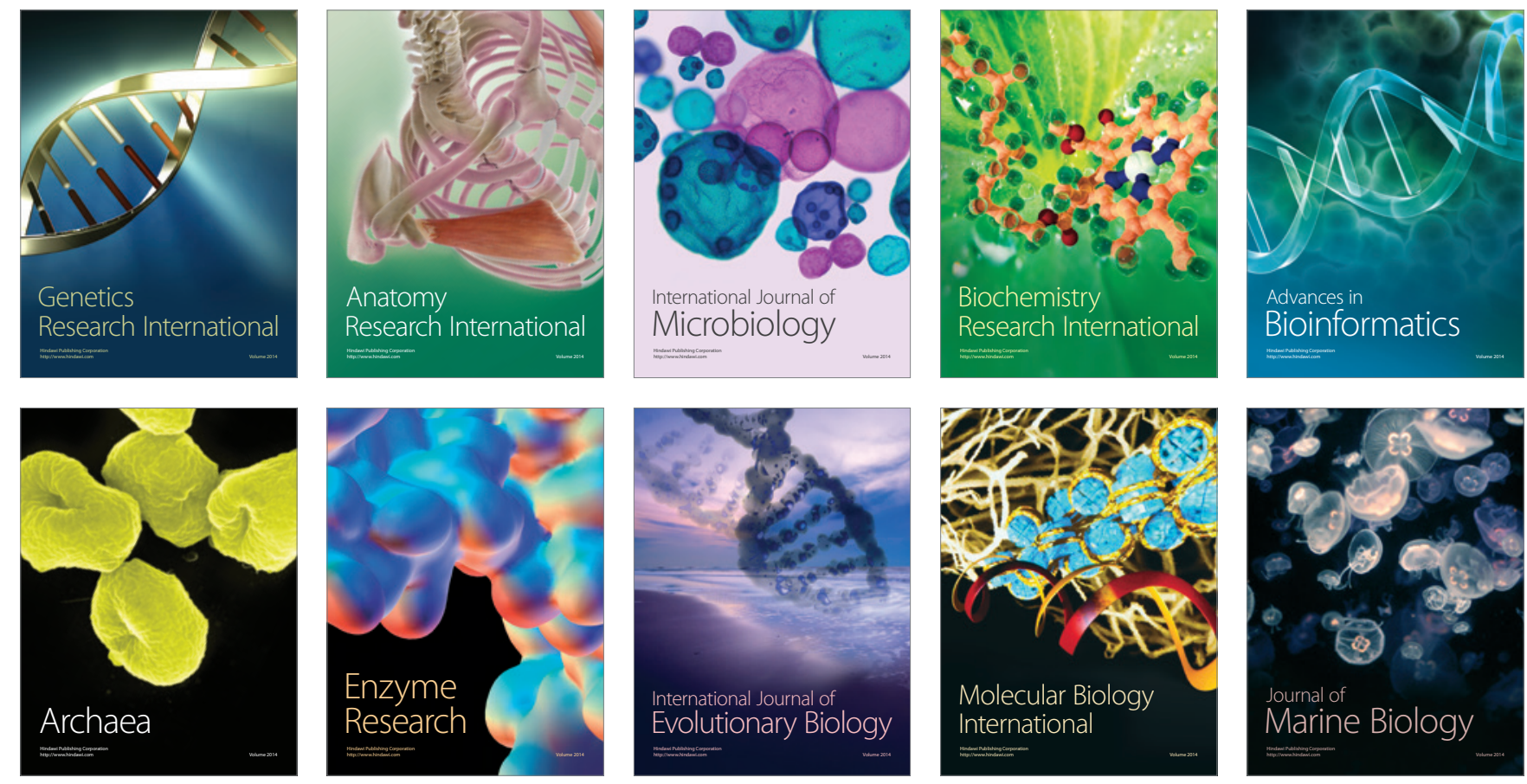